\title{
An improved Recursive Construction for Disjoint Steiner Quadruple Systems
}

\author{
Tuvi Etzion* Junling Zhou ${ }^{\dagger}$
}

December 11, 2019

\begin{abstract}
Let $D(n)$ be the number of pairwise disjoint Steiner quadruple systems. A simple counting argument shows that $D(n) \leq n-3$ and a set of $n-3$ such systems is called a large set. No nontrivial large set was constructed yet, although it is known that they exist if $n \equiv 2$ or $4(\bmod 6)$ is large enough. When $n \geq 7$ and $n \equiv 1$ or $5(\bmod 6)$, we present a recursive construction and prove a recursive formula on $D(4 n)$, as follows:

$$
D(4 n) \geq 2 n+\min \{D(2 n), 2 n-7\} .
$$

The related construction has a few advantages over some of the previously known constructions for pairwise disjoint Steiner quadruple systems.
\end{abstract}

Keywords: Disjoint Steiner systems, large set, Latin square, one-factor, one-factorization, Steiner quadruple system.

\footnotetext{
*Department of Computer Science, Technion, Haifa 3200003, Israel, e-mail: etzion@cs.technion.ac.il.

$\dagger^{\dagger}$ Department of Mathematics, Beijing Jiaotong University, Beijing, China, e-mail: jlzhou@bjtu.edu.cn.

${ }^{1}$ This research was supported in part by the 111 Project of China (B16002) and in part by the NSFC grants 11571034 and 11971053. Part of the research was performed during a visit of T. Etzion to Beijing Jiaotong University. He expresses sincere thanks to the 111 Project of China (B16002) for its support and to the Department of Mathematics at Beijing Jiaotong University for their kind hospitality.
} 


\section{Introduction}

A Steiner system of order $n, S(t, k, n)$, is a pair $(Q, B)$, where $Q$ is an $n$-set (whose elements are called points) and $B$ is a collection of $k$-subsets (called blocks) of $Q$, such that each $t$-subset of $Q$ is contained in exactly one block of $B$.

A large set of Steiner systems $S(t, k, n)$, on an $n$-set $Q$, is a partition of all $k$-subsets of $Q$ into Steiner systems $S(t, k, n)$. If we restrict ourself to Steiner systems $S(t-1, t, n)$, then exactly two families of large sets are solved completely. A Steiner system $S(1,2, n)$ exists if and only if $n$ is even and its large set is known as a one-factorization of the complete graph $K_{n}$. The existence of such one-factorizations is a folklore and a survey can be found in [17]. A Steiner system $S(2,3, n)$ is known as Steiner triple system and the corresponding large set is known to exist for every admissible $n \equiv 1$ or $3(\bmod 6)$, where $n>7$. It was first proved by Lu [12, 13], who left six open cases which were solved by Teirlinck [16]. An alternative shorter proof was given later by Ji [6].

A Steiner system $S(3,4, n)$ is also called a Steiner quadruple system of order $n$ and it is denoted by $\operatorname{SQS}(n)$. These systems which are of special interest exist if and only if $n \equiv 2$ or $4(\bmod 6)[5]$. No construction of nontrivial large set of $\operatorname{SQS}(n)$ is known, although it is a common belief that they exist even for small $n$. Using probabilistic arguments, their existence for large enough $n$, $n \equiv 2$ or $4(\bmod 6)$, was proved recently [7]. In practice, it is of interest to construct many disjoint systems. Let $D(n)$ be the number of pairwise disjoint $\mathrm{SQS}(n) \mathrm{s}$ (PDQs of order $n$ in short, the order is omitted when it is understood). The size of an $\operatorname{SQS}(n)$ is $\left(\begin{array}{l}n \\ 3\end{array}\right) / 4$, and hence $D(n) \leq n-3$ and a large set contains $n-3$ PDQs of order $n$, i.e. $D(n)=n-3$, if a large set exists.

The first lower bound on $D(n)$ was given by Lindner [10] who proved that if $n \equiv 2$ or $4(\bmod 6)$, then $D(2 n) \geq n$. He further obtained another bound [11], $D(4 n) \geq 3 n$ if $n \equiv 2$ or $4(\bmod 6)$, where $n \geq 8$. In a sequence of papers [9, 14, 15] it was proved that $D(2 n) \geq n$ for $n=5^{a} 13^{b} 17^{c}$, where $a, b, c \geq 0$ and $a+b+c>0$ and for $n=11$. Etzion and Hartman [3] has proved that $D\left(2^{k} n\right) \geq\left(2^{k}-1\right) n, k \geq 2$, if there exists a set of $3 n$ PDQs of order $4 n$ with a certain structure. The result can be applied on $n=11, n \equiv 2$ or $4(\bmod 6)$, and $n=5^{a} 13^{b} 17^{c}$, where $a, b, c \geq 0$ and $a+b+c>0$. In particular it is proved in [3] that $D\left(5 \cdot 2^{m}\right) \geq 5 \cdot 2^{m}-5$, which falls short by just one system from a large set. This claim follows from the fact that $5 \cdot 2^{m}-4$ PDQs imply that the remaining quadruples which are not contained in these PDQs form another SQS $\left(5 \cdot 2^{m}\right)$. The construction in [3] is recursive, based on the existence of 2-chromatic PDQs. A recursive equation for $D(4 n), n \equiv 1$ or $5(\bmod 6)$, was given in [1], where it is proved that

$$
D(4 n) \geq 2 n+\min \{D(2 n), n-2\} .
$$

Our goal in this paper is to improve this recursive formula to the following theorem which is the main result of the paper.

Theorem 1. If $n \geq 7$ and $n \equiv 1$ or $5(\bmod 6)$, then $D(4 n) \geq 2 n+\min \{D(2 n), 2 n-7\}$.

The rest of this paper is organized as follows. In Section 2 we present the two known recursive doubling construction for SQSs and for a set of PDQs. The proof of Theorem 1 is given in Section 3 with its related construction. We start with any $\operatorname{SQS}(2 n)$, a certain Latin square of order $2 n$, and apply the first doubling construction (DLS Construction) to obtain $2 n$ PDQs of order $4 n$. We analyse the quadruples which are contained in the constructed $2 n$ PDQs and continue with the second doubling construction (DB Construction) to form PDQs of order $4 n$ which contain 
quadruples which are not contained in the first $2 n$ PDQs. The construction for $n \equiv 5(\bmod 6)$ is slightly simpler to explain than the one for $n \equiv 1(\bmod 6)$ and also when $n$ is a prime some of the construction is slightly simpler. We make some distinction between these cases in the proofs. Examples of each step are presented throughout the description of the related recursive construction. In Section 4 a conclusion, comparison with previous results, and several problems for future research, are presented.

\section{Two Recursive Constructions}

In this section we will discuss two main recursive doubling constructions for SQSs or more precisely, for a set of PDQs. The first one is a doubling construction due to Lindner [10]. It will be called DLS (for Doubling Lindner Systems) Construction. We will present a slightly different variant from the one given in [10]. This variant was already presented in [2]. The second construction is a folklore doubling construction which will be called DB (for doubling) Construction. These two constructions will be the main ingredients for the other constructions presented in this paper.

For the two constructions we need to define several well-known combinatorial designs which are essential in the construction used to prove Theorem 1 in Section 3 ,

A $v \times v$ Latin square is a $v \times v$ array in which each row and each column is a permutation of a $v$-set $Q$. A $k \times v$ array is called a Latin rectangle if each row is a permutation of $Q$ and each column contains $k$ distinct elements. It is well known that such a rectangle can be completed to an $v \times v$ Latin square, for example by applying the well-known Hall's marriage theorem [4]. A Latin square has no $2 \times 2$ subsquares if any $2 \times 2$ subsquare restricted to two rows and two columns does not form a Latin square [8].

A one-factorization $F=\left\{F_{0}, F_{1}, \ldots, F_{v-2}\right\}$ of the complete graph $K_{v}$, where $v$ is an even positive integer, is partition of the edges of $K_{v}$ into perfect matchings. Each $F_{i}, 0 \leq i \leq v-2$, is a perfect matching of $K_{v}$, which is also called a one-factor. As mentioned before a one-factor is a Steiner system $S(1,2, v)$ and a one-factorization is a related large set. Clearly, a one-factor contains $\frac{v}{2}$ pairs of vertices whose union is the set of $v$ vertices in $K_{v}$.

\subsection{DLS Construction}

Let $\left(\mathbb{Z}_{v}, B\right)$ be an $\operatorname{SQS}(v)$ and let $A$ be a $v \times v$ Latin square of order $v$, on the point set $\mathbb{Z}_{v}$, with no $2 \times 2$ subsquares. Denote by $\alpha_{i}$ the permutation on $\mathbb{Z}_{v}$ defined by $\alpha_{i}(j)=y$ if and only if $A(i, j)=y$. For each $i, 0 \leq i \leq v-1$, we define a set of quadruples $B_{i}$ on $\mathbb{Z}_{v} \times \mathbb{Z}_{2}$ as follows:

1. For each quadruple $\left\{x_{1}, x_{2}, x_{3}, x_{4}\right\} \in B$, the following 8 quadruples are contained in $B_{i}$.

$$
\begin{array}{lll}
\left\{\left(x_{1}, 0\right),\left(x_{2}, 0\right),\left(x_{3}, 0\right),\left(\alpha_{i}\left(x_{4}\right), 1\right)\right\}, & \left\{\left(x_{1}, 1\right),\left(x_{2}, 1\right),\left(x_{3}, 1\right),\left(\alpha_{i}\left(x_{4}\right), 0\right)\right\} \\
\left\{\left(x_{1}, 0\right),\left(x_{2}, 0\right),\left(\alpha_{i}\left(x_{3}\right), 1\right),\left(x_{4}, 0\right)\right\}, & \left\{\left(x_{1}, 1\right),\left(x_{2}, 1\right),\left(\alpha_{i}\left(x_{3}\right), 0\right),\left(x_{4}, 1\right)\right\} \\
\left\{\left(x_{1}, 0\right),\left(\alpha_{i}\left(x_{2}\right), 1\right),\left(x_{3}, 0\right),\left(x_{4}, 0\right)\right\}, & \left\{\left(x_{1}, 1\right),\left(\alpha_{i}\left(x_{2}\right), 0\right),\left(x_{3}, 1\right),\left(x_{4}, 1\right)\right\} \\
\left\{\left(\alpha_{i}\left(x_{1}\right), 1\right),\left(x_{2}, 0\right),\left(x_{3}, 0\right),\left(x_{4}, 0\right)\right\}, & \left\{\left(\alpha_{i}\left(x_{1}\right), 0\right),\left(x_{2}, 1\right),\left(x_{3}, 1\right),\left(x_{4}, 1\right)\right\}
\end{array}
$$

2. For each pair $\left\{x_{1}, x_{2}\right\} \subset \mathbb{Z}_{v}$, the quadruple $\left\{\left(x_{1}, 0\right),\left(x_{2}, 0\right),\left(\alpha_{i}\left(x_{1}\right), 1\right),\left(\alpha_{i}\left(x_{2}\right), 1\right)\right\}$ is contained in $B_{i}$.

This DLS Construction is a variant [2] of the Lindner Construction [10]. It yields $v$ PDQs of order $2 v$. 


\subsection{DB Construction}

Let $\left(\mathbb{Z}_{v}, B\right)$ be an $\operatorname{SQS}(v)$, let $F=\left\{F_{0}, F_{1}, \ldots, F_{v-2}\right\}$ and $F^{\prime}=\left\{F_{0}^{\prime}, F_{1}^{\prime}, \ldots, F_{n-2}^{\prime}\right\}$ be two onefactorizations of $K_{v}$ on the vertex set $\mathbb{Z}_{v}$, and let $\alpha$ be any permutation on the set $\{0,1, \ldots, v-2\}$. We define a collection of quadruples $B^{\prime}$ on $\mathbb{Z}_{v} \times \mathbb{Z}_{2}$ as follows.

1. For each quadruple $\left\{x_{1}, x_{2}, x_{3}, x_{4}\right\} \in B$, the following two quadruples are contained in $B^{\prime}$.

$$
\left\{\left(x_{1}, 0\right),\left(x_{2}, 0\right),\left(x_{3}, 0\right),\left(x_{4}, 0\right)\right\}, \quad\left\{\left(x_{1}, 1\right),\left(x_{2}, 1\right),\left(x_{3}, 1\right),\left(x_{4}, 1\right)\right\} .
$$

2. For each $i \in\{0,1, \ldots, n-2\}$ and $\left\{x_{1}, x_{2}\right\} \in F_{i},\left\{y_{1}, y_{2}\right\} \in F_{j}^{\prime}$, where $j=\alpha(i)$, the following quadruple is contained in $B^{\prime}$.

$$
\left\{\left(x_{1}, 0\right),\left(x_{2}, 0\right),\left(y_{1}, 1\right),\left(y_{2}, 1\right)\right\} .
$$

The set of all such quadruples produced by $F_{i}$ and $F_{j}^{\prime}$ are said to be the direct product of $F_{i}$ and $F_{j}^{\prime}$. For the construction in Section 3, it is assumed that instead of one $\operatorname{SQS}(v) B$, we have a set of $k$ PDQs of order $v$ and instead of $\alpha$ we use $k$ permutations on $\{0,1, \ldots, v-2\}$ defined by the rows of a $k \times(v-1)$ Latin rectangle. In this case, the DB construction yields a set of $k$ PDQs of order $2 v$.

\subsection{Configurations}

Both constructions, the DLS Construction and the DB Construction, are based on a partition of the point set $Q$ into two equal sets, say $P_{1}$ and $P_{2}$. Given such a partition and a quadruple $X=\left\{x_{1}, x_{2}, x_{3}, x_{4}\right\}$ of $Q$, we say that $X$ is a 4 -subset from configuration $(i, j)$, where $i+j=4$, if $\left|X \cap P_{1}\right|=i$ and $\left|X \cap P_{2}\right|=j$. There are clearly five possible configurations, $(4,0),(3,1),(2,2)$, $(1,3)$, and $(0,4)$. The following trivial lemma can be easily verified.

Lemma 1. In the DLS construction, based on the partition of $\mathbb{Z}_{v} \times\{0,1\}$ into $\mathbb{Z}_{v} \times\{0\}$ and $\mathbb{Z}_{n} \times\{1\}$, each quadruple from configuration $(3,1)$ and each quadruple from configuration $(1,3)$ is contained exactly once in the $v$ PDQs of order $2 v$.

\section{An Improved Recursive Construction}

In this section a recursive doubling construction for PDQs of order $4 n$, where $n$ is an odd integer not divisible by 3 , is presented. The construction proceeds in two parts, where we employ the two doubling constructions with $v=2 n, n \equiv 1$ or $5(\bmod 6)$. In the first part the DLS Construction is applied with an appropriate $(2 n) \times(2 n)$ Latin square. A set of $2 n$ PDQs of order $4 n$ is obtained in this part. By Lemma 1, each quadruple from configurations $(3,1)$ and each quadruple from configuration $(1,3)$ is contained in these $2 n$ PDQs. We analyse the quadruples from configuration $(2,2)$ contained in these $2 n$ PDQs. In the second part, the DB Construction is applied with a set of $k$ PDQs of order $2 n$ and a $k \times(2 n-1)$ Latin rectangle which yields quadruples from configuration $(2,2)$ which are not obtained in the first part of the construction. The final outcome from the two parts of the construction are $2 n+k$ PDQs of order $4 n$ which will imply Theorem 1. 


\subsection{PDQs from the DLS Construction}

The first step in the first part of the construction is to define a $(2 n) \times(2 n)$ Latin square to be used in the DLS Construction. This Latin square was defined in [8] and used in [1] for constructions of PDQs of order $4 n$. Let $A_{n}, B_{n}$, be the two $n \times n$ Latin squares defined as follows.

$$
\begin{gathered}
A_{n}(i, j) \equiv i+j-2(\bmod n), \quad 1 \leq i, j \leq n, \\
B_{n}(i, j) \equiv i-j(\bmod n), \quad 1 \leq i, j \leq n,
\end{gathered}
$$

where the values in $A_{n}$ and $B_{n}$ are reduced to the range $\{0,1, \ldots, n-1\}$.

Let $C_{n}$ and $D_{n}$ be the following two $n \times n$ Latin squares, where for each $0 \leq i, j \leq n-1$, we have

$$
\begin{gathered}
C_{n}(i, j)=A_{n}(i, j) \text { reduced modulo } n \text { to the range }\{n, n+1, \ldots, 2 n-1\}, \\
D_{n}(i, j)=A_{n}(i, j)-1 \text { reduced modulo } n \text { to the range }\{n, n+1, \ldots, 2 n-1\} .
\end{gathered}
$$

The $(2 n) \times(2 n)$ Latin square $M_{n}$ which will be used in the DLS Construction is given by

$$
M_{n}=\left[\begin{array}{cc}
B_{n} & C_{n} \\
D_{n} & B_{n}
\end{array}\right]
$$

It was proved in [8] that $M_{n}$ has no $2 \times 2$ subsquares.

Example 1. Throughout this section, we will concentrate on a few examples, for $n=7, n=11$, an also $n=19$.

For $n=7$, the $14 \times 14$ Latin square $M_{7}$ is given by

$$
\left[\begin{array}{cccccccccccccc}
0 & 6 & 5 & 4 & 3 & 2 & 1 & 7 & 8 & 9 & 10 & 11 & 12 & 13 \\
1 & 0 & 6 & 5 & 4 & 3 & 2 & 8 & 9 & 10 & 11 & 12 & 13 & 7 \\
2 & 1 & 0 & 6 & 5 & 4 & 3 & 9 & 10 & 11 & 12 & 13 & 7 & 8 \\
3 & 2 & 1 & 0 & 6 & 5 & 4 & 10 & 11 & 12 & 13 & 7 & 8 & 9 \\
4 & 3 & 2 & 1 & 0 & 6 & 5 & 11 & 12 & 13 & 7 & 8 & 9 & 10 \\
5 & 4 & 3 & 2 & 1 & 0 & 6 & 12 & 13 & 7 & 8 & 9 & 10 & 11 \\
6 & 5 & 4 & 3 & 2 & 1 & 0 & 13 & 7 & 8 & 9 & 10 & 11 & 12 \\
& & & & & & & & & & & & & \\
13 & 7 & 8 & 9 & 10 & 11 & 12 & 0 & 6 & 5 & 4 & 3 & 2 & 1 \\
7 & 8 & 9 & 10 & 11 & 12 & 13 & 1 & 0 & 6 & 5 & 4 & 3 & 2 \\
8 & 9 & 10 & 11 & 12 & 13 & 7 & 2 & 1 & 0 & 6 & 5 & 4 & 3 \\
9 & 10 & 11 & 12 & 13 & 7 & 8 & 3 & 2 & 1 & 0 & 6 & 5 & 4 \\
10 & 11 & 12 & 13 & 7 & 8 & 9 & 4 & 3 & 2 & 1 & 0 & 6 & 5 \\
11 & 12 & 13 & 7 & 8 & 9 & 10 & 5 & 4 & 3 & 2 & 1 & 0 & 6 \\
12 & 13 & 7 & 8 & 9 & 10 & 11 & 6 & 5 & 4 & 3 & 2 & 1 & 0
\end{array}\right]
$$




\subsection{PDQs from the DB Construction}

Now, we apply the DB Construction and obtain PDQs which are also disjoint from the ones obtained in the first part via the DLS Construction. To this end, we have first to analyse the quadruples from configuration $(2,2)$ which are contained in the $2 n$ PDQs obtained in the DLS Construction. The reason is that by Lemma 1 we can ignore quadruples from configuration $(3,1)$ and configuration $(1,3)$ and clearly no quadruple from configurations $(4,0)$ and $(0,4)$ is obtained in the DLS construction.

\subsubsection{Quadruples from Configuration $(2,2)$ in the DLS Construction}

The quadruples from configuration $(2,2)$ which are contained in the $2 n$ PDQs of order $4 n$ from the DLS Construction can be defined by observing the properties of the Latin square $M_{n}$. First, note that four sets of pairs are defined.

$$
\begin{gathered}
\mathcal{A}_{i} \triangleq\left\{\{x, y\}: x, y \in \mathbb{Z}_{n}, y-x \equiv i(\bmod n)\right\}, \quad 1 \leq i \leq \frac{n-1}{2}, \\
\mathcal{B}_{i} \triangleq\{\{x, y\}: x, y \in\{n, n+1, \ldots, 2 n-1\}, y-x \equiv i(\bmod n)\}, \quad 1 \leq i \leq \frac{n-1}{2}, \\
\mathcal{C}_{i} \triangleq\{\{x, y\}: 0 \leq x \leq n-1, n \leq y \leq 2 n-1, x+y \equiv i(\bmod n)\}, \quad 0 \leq i \leq n-1, \\
\mathcal{D}_{i} \triangleq\{\{x, y\}: 0 \leq x \leq n-1, n \leq y \leq 2 n-1, y-x \equiv i(\bmod n)\}, \quad 0 \leq i \leq n-1 .
\end{gathered}
$$

Example 2. For $n=7$, the sets $\mathcal{A}_{i}$ 's, $\mathcal{B}_{i}$ 's, $\mathcal{C}_{i}$ 's, and $\mathcal{D}_{i}$ 's, are as follows.

$$
\begin{gathered}
\mathcal{A}_{1}=\{\{0,1\},\{1,2\},\{2,3\},\{3,4\},\{4,5\},\{5,6\},\{6,0\}\} \\
\mathcal{A}_{2}=\{\{0,2\},\{1,3\},\{2,4\},\{3,5\},\{4,6\},\{5,0\},\{6,1\}\} \\
\mathcal{A}_{3}=\{\{0,3\},\{1,4\},\{2,5\},\{3,6\},\{4,0\},\{5,1\},\{6,2\}\} \\
\mathcal{B}_{1}=\{\{7,8\},\{8,9\},\{9,10\},\{10,11\},\{11,12\},\{12,13\},\{13,7\}\} \\
\mathcal{B}_{2}=\{\{7,9\},\{8,10\},\{9,11\},\{10,12\},\{11,13\},\{12,7\},\{13,8\}\} \\
\mathcal{B}_{3}=\{\{7,10\},\{8,11\},\{9,12\},\{10,13\},\{11,7\},\{12,8\},\{13,9\}\} \\
\mathcal{C}_{0}=\{\{0,7\},\{1,13\},\{2,12\},\{3,11\},\{4,10\},\{5,9\},\{6,8\}\} \\
\mathcal{C}_{1}=\{\{0,8\},\{1,7\},\{2,13\},\{3,12\},\{4,11\},\{5,10\},\{6,9\}\} \\
\mathcal{C}_{2}=\{\{0,9\},\{1,8\},\{2,7\},\{3,13\},\{4,12\},\{5,11\},\{6,10\}\} \\
\mathcal{C}_{3}=\{\{0,10\},\{1,9\},\{2,8\},\{3,7\},\{4,13\},\{5,12\},\{6,11\}\} \\
\mathcal{C}_{4}=\{\{0,11\},\{1,10\},\{2,9\},\{3,8\},\{4,7\},\{5,13\},\{6,12\}\} \\
\mathcal{C}_{5}=\{\{0,12\},\{1,11\},\{2,10\},\{3,9\},\{4,8\},\{5,7\},\{6,13\}\} \\
\mathcal{C}_{6}=\{\{0,13\},\{1,12\},\{2,11\},\{3,10\},\{4,9\},\{5,8\},\{6,7\}\}
\end{gathered}
$$




$$
\begin{aligned}
& \mathcal{D}_{0}=\{\{0,7\},\{1,8\},\{2,9\},\{3,10\},\{4,11\},\{5,12\},\{6,13\}\} \\
& \mathcal{D}_{1}=\{\{0,8\},\{1,9\},\{2,10\},\{3,11\},\{4,12\},\{5,13\},\{6,7\}\} \\
& \mathcal{D}_{2}=\{\{0,9\},\{1,10\},\{2,11\},\{3,12\},\{4,13\},\{5,7\},\{6,8\}\} \\
& \mathcal{D}_{3}=\{\{0,10\},\{1,11\},\{2,12\},\{3,13\},\{4,7\},\{5,8\},\{6,9\}\} \\
& \mathcal{D}_{4}=\{\{0,11\},\{1,12\},\{2,13\},\{3,7\},\{4,8\},\{5,9\},\{6,10\}\} \\
& \mathcal{D}_{5}=\{\{0,12\},\{1,13\},\{2,7\},\{3,8\},\{4,9\},\{5,10\},\{6,11\}\} \\
& \mathcal{D}_{6}=\{\{0,13\},\{1,7\},\{2,8\},\{3,9\},\{4,10\},\{5,11\},\{6,12\}\}
\end{aligned}
$$

The following two lemmas are immediate observations from the definitions of the $\mathcal{A}_{i}$ 's, $\mathcal{B}_{i}$ 's, $\mathcal{C}_{i}{ }^{\prime}$ s, $\mathcal{D}_{i}$ 's, and $M_{n}$.

Lemma 2. By using the Latin square $M_{n}$, the following sets of quadruples are contained, in the first $2 n$ PDQs obtained via the DLS Construction.

$$
\begin{gathered}
\{(v, 0),(x, 0),(y, 1),(z, 1)\}, \quad\{v, x\} \in \mathcal{A}_{i} \cup \mathcal{B}_{i}, \quad\{y, z\} \in \mathcal{A}_{i} \cup \mathcal{B}_{i}, 1 \leq i \leq \frac{n-1}{2}, \\
\{(v, 0),(x, 0),(y, 1),(z, 1)\}, \quad\{v, x\} \in \mathcal{C}_{i}, \quad\{y, z\} \in \mathcal{D}_{i-1} \cup \mathcal{D}_{i}, 0 \leq i \leq n-1,
\end{gathered}
$$

where subscripts are taken modulo $n$. Each one of these quadruples is contained in exactly one of these $2 n$ PDQs. No other quadruple from configuration $(2,2)$ is contained in these $2 n$ PDQs.

Note, that Lemma 22 implies that the quadruples from configuration $(2,2)$ formed by the first $2 n$ PDQs are of the form $\{(v, 0),(x, 0),(y, 1),(z, 1)\}$. The pair $\{v, x\}$ is from one of the $2 n-1$ sets $\mathcal{A}_{i}, \mathcal{B}_{i}, 1 \leq i \leq \frac{n-1}{2}$, or $\mathcal{C}_{j}, 0 \leq j \leq n-1$. The pair $\{y, z\}$ is from one of the $2 n-1$ sets $\mathcal{A}_{i}$, $\mathcal{B}_{i}, 1 \leq i \leq \frac{n-1}{2}$, or $\mathcal{D}_{j}, 0 \leq j \leq n-1$. These facts will be used in the sequel. Now, we state the second simple lemma which can be easily verified.

\section{Lemma 3.}

1. Each one of the $\mathcal{C}_{i}$ 's and the $\mathcal{D}_{i}$ 's is a one-factor of $K_{2 n}$.

2. $\bigcup_{i=1}^{(n-1) / 2}\left(\mathcal{A}_{i} \cup \mathcal{B}_{i}\right) \cup \bigcup_{i=0}^{n-1} \mathcal{C}_{i}=\{\{x, y\}: 0 \leq x<y \leq 2 n-1\}$.

3. $\bigcup_{i=1}^{(n-1) / 2}\left(\mathcal{A}_{i} \cup \mathcal{B}_{i}\right) \cup \bigcup_{i=0}^{n-1} \mathcal{D}_{i}=\{\{x, y\}: 0 \leq x<y \leq 2 n-1\}$.

4. $\sum_{i=1}^{(n-1) / 2}\left(\left|\mathcal{A}_{i}\right|+\left|\mathcal{B}_{i}\right|\right)+\sum_{i=0}^{n-1}\left|\mathcal{C}_{i}\right|=n(2 n-1)$.

5. $\sum_{i=1}^{(n-1) / 2}\left(\left|\mathcal{A}_{i}\right|+\left|\mathcal{B}_{i}\right|\right)+\sum_{i=0}^{n-1}\left|\mathcal{D}_{i}\right|=n(2 n-1)$. 


\subsubsection{One-Factorizations for the DB Construction}

The second part of the construction is to use the DB Construction to form more PDQs or order $4 n$ with quadruples which were not used in the first $2 n$ PDQs. Such a set of new PDQs of order $4 n$ depends on the value of $D(2 n)$. These PDQs of order $2 n$ will form the quadruples from configuration $(4,0)$ and from configuration $(0,4)$ in the recursive construction. We distinguish now between the case $n \equiv 5(\bmod 6)$ and the case $n \equiv 1(\bmod 6)$.

Lemma 4 . Let $n \equiv 1$ or $5(\bmod 6)$ be a prime. Then there exists a one-factorization $\mathcal{R}$ of $K_{2 n}$ on the vertex set $\{0,1, \ldots, 2 n-1\}$ satisfying the followings:

1. if $n \equiv 5(\bmod 6)$, then $\mathcal{R}=\left\{\mathcal{R}_{i, j}: 1 \leq i \leq \frac{2 n-1}{3}, 1 \leq j \leq 3\right\}$, where

$$
\mathcal{R}_{i, 1} \cup \mathcal{R}_{i, 2} \cup \mathcal{R}_{i, 3}=\mathcal{A}_{i} \cup \mathcal{B}_{i} \cup \mathcal{C}_{i-1}, 1 \leq i \leq \frac{n-1}{2},
$$

and

$$
\mathcal{R}_{i, 1}=\mathcal{C}_{3 i-n-2}, \mathcal{R}_{i, 2}=\mathcal{C}_{3 i-n-1}, \mathcal{R}_{i, 3}=\mathcal{C}_{3 i-n}, \frac{n+1}{2} \leq i \leq \frac{2 n-1}{3} .
$$

2. if $n \equiv 1(\bmod 6)$, then $\mathcal{R}=\left\{\mathcal{R}_{i, j}: 1 \leq i \leq \frac{n-1}{2}, 1 \leq j \leq 3\right\} \cup\left\{\mathcal{C}_{i}: \frac{n-1}{2} \leq i \leq n-1\right\}$, where Eq. (1) also holds.

Proof. By Lemma 3, each $\mathcal{C}_{i}$ is a one-factor of $K_{2 n}$ and $\bigcup_{i=1}^{\frac{n-1}{2}}\left(\mathcal{A}_{i} \cup \mathcal{B}_{i}\right) \cup\left(\bigcup_{i=0}^{n-1} \mathcal{C}_{i}\right)$ covers all pairs of $\{0,1, \ldots, 2 n-1\}$. So, to prove the conclusion, we only need to show that there exist one-factors $\mathcal{R}_{i, j}$ with $1 \leq i \leq \frac{2 n-1}{3}$ and $j=1,2,3$ such that Eq. (11) holds.

For a given $1 \leq i \leq \frac{n-1}{2}$, form the sequences $s_{j}=\{j \cdot i,(j+1) \cdot i\}, 0 \leq j \leq n-1$, where the elements are taken modulo $n$, reduced to the range $\{0,1, \ldots, n-1\}$. Form the second sequence $q_{j}=\{j \cdot i,(j+1) \cdot i\}, 0 \leq j \leq n-1$, where elements are taken modulo $n$, reduced to the range $\{n, n+1, \ldots, 2 n-1\}$. It is obvious that $\mathcal{A}_{i}=\left\{s_{j}: 0 \leq j \leq n-1\right\}$ and $\mathcal{B}_{i}=\left\{q_{j}: 0 \leq j \leq n-1\right\}$. Since $n$ is prime, the $n$ pairs of $\mathcal{A}_{i}$ form an $n$-cycle (a cycle of length $n$ ), so are those of $\mathcal{B}_{i}$. Note that $s_{0}=\{0, i\} \in \mathcal{A}_{i}, q_{m}=\{n+i-1,2 n-1\} \in \mathcal{B}_{i}$ where $n-1 \equiv m \cdot i(\bmod n)(0 \leq m \leq n-1)$. Since $n$ is odd, applying the $n$-cycle of $\mathcal{A}_{i}$ we can partition $\mathcal{A}_{i} \backslash\left\{s_{0}\right\}$ into two subsets $\mathcal{A}_{i, 1}$ and $\mathcal{A}_{i, 2}$ with equal size $\frac{n-1}{2}$ and

$$
\bigcup_{P \in \mathcal{A}_{i, 1}} P=\{0,1, \ldots, n-1\} \backslash\{0\}, \bigcup_{P \in \mathcal{A}_{i, 2}} P=\{0,1, \ldots, n-1\} \backslash\{i\} .
$$

Similarly, we can partition $\mathcal{B}_{i} \backslash\left\{q_{m}\right\}$ into two subsets $\mathcal{B}_{i, 1}$ and $\mathcal{B}_{i, 2}$ with equal size $\frac{n-1}{2}$ and

$$
\bigcup_{P \in \mathcal{B}_{i, 1}} P=\{n, n+1, \ldots, 2 n-1\} \backslash\{n+i-1\}, \bigcup_{P \in \mathcal{B}_{i, 2}} P=\{n, n+1, \ldots, 2 n-1\} \backslash\{2 n-1\} .
$$

Obviously we have $\{0, n+i-1\},\{i, 2 n-1\} \in \mathcal{C}_{i-1}$. So define

$$
\begin{aligned}
& \mathcal{R}_{i, 1}=\mathcal{A}_{i, 1} \cup \mathcal{B}_{i, 1} \cup\{\{0, n+i-1\}\}, \\
& \mathcal{R}_{i, 2}=\mathcal{A}_{i, 2} \cup \mathcal{B}_{i, 2} \cup\{\{i, 2 n-1\}\}, \\
& \mathcal{R}_{i, 3}=\left(\mathcal{C}_{i-1} \cup\left\{s_{0}, q_{m}\right\}\right) \backslash\{\{0, n+i-1\},\{i, 2 n-1\}\} .
\end{aligned}
$$

It is easy to verify that each $\mathcal{R}_{i, j}$ is a one-factor $\left(1 \leq i \leq \frac{2 n-1}{3}\right.$ and $\left.1 \leq j \leq 3\right)$ and Eq. (11) holds. This completes the proof. 
A related result will be given in the following lemma for a one-factorization $\mathcal{T}$ with almost an identical proof.

Lemma 5. Let $n \equiv 1$ or $5(\bmod 6)$ be a prime. Then there exists a one-factorization $\mathcal{T}$ of $K_{2 n}$ on the vertex set $\{0,1, \ldots, 2 n-1\}$ satisfying the followings:

1. if $n \equiv 5(\bmod 6)$, then $\mathcal{T}=\left\{\mathcal{T}_{i, j}: 1 \leq i \leq \frac{2 n-1}{3} 1 \leq j \leq 3\right\}$, where

$$
\mathcal{T}_{i, 1} \cup \mathcal{T}_{i, 2} \cup \mathcal{T}_{i, 3}=\mathcal{A}_{i} \cup \mathcal{B}_{i} \cup \mathcal{D}_{i-1}, 1 \leq i \leq \frac{n-1}{2},
$$

and

$$
\mathcal{T}_{i, 1}=\mathcal{D}_{3 i-n-2}, \mathcal{T}_{i, 2}=\mathcal{D}_{3 i-n-1}, \mathcal{T}_{i, 3}=\mathcal{D}_{3 i-n}, \frac{n+1}{2} \leq i \leq \frac{2 n-1}{3} .
$$

2. if $n \equiv 1(\bmod 6)$, then $\mathcal{T}=\left\{\mathcal{T}_{i, j}: 1 \leq i \leq \frac{n-1}{21 \leq j \leq 3}\right\} \cup\left\{\mathcal{D}_{i}: \frac{n-1}{2} \leq i \leq n-1\right\}$, where Eq. (2) also holds.

Proof. The proof is very similar to that of Lemma 4. The difference is that $\mathcal{D}_{i}$ is used instead of $\mathcal{C}_{i}$. To prove that, for $1 \leq i \leq \frac{n-1}{2}, \mathcal{A}_{i} \cup \mathcal{B}_{i} \cup \mathcal{D}_{i-1}$ can be partitioned into three one-factors $\mathcal{T}_{i, 1}, \mathcal{T}_{i, 2}$, and $\mathcal{T}_{i, 3}$, we note that $\{0, i\} \in \mathcal{A}_{i},\{n+i-1, n+2 i-1\} \in \mathcal{B}_{i}$ and $\{0, n+i-1\},\{i, n+2 i-1\} \in \mathcal{D}_{i-1}$. The rest of the proof is exactly parallel to that of Lemma 4 .

Example 3. For $n=11$, the set $\mathcal{R}$ and part of the set $\mathcal{T}$ are given by the following sets.

$$
\begin{aligned}
& \mathcal{R}_{1,1}=\{\{1,2\},\{3,4\},\{5,6\},\{7,8\},\{9,10\},\{12,13\},\{14,15\},\{16,17\},\{18,19\},\{20,21\},\{0,11\}\} \\
& \mathcal{R}_{1,2}=\{\{2,3\},\{4,5\},\{6,7\},\{8,9\},\{10,0\},\{11,12\},\{13,14\},\{15,16\},\{17,18\},\{19,20\},\{1,21\}\} \\
& \mathcal{R}_{1,3}=\{\{2,20\},\{3,19\},\{4,18\},\{5,17\},\{6,16\},\{7,15\},\{8,14\},\{9,13\},\{10,12\},\{0,1\},\{11,21\}\} \\
& \mathcal{R}_{2,1}=\{\{2,4\},\{3,5\},\{6,8\},\{7,9\},\{1,10\},\{11,13\},\{14,16\},\{15,17\},\{18,20\},\{19,21\},\{0,12\}\} \\
& \mathcal{R}_{2,2}=\{\{1,3\},\{4,6\},\{5,7\},\{8,10\},\{0,9\},\{12,14\},\{13,15\},\{16,18\},\{17,19\},\{11,20\},\{2,21\}\} \\
& \mathcal{R}_{2,3}=\{\{1,11\},\{3,20\},\{4,19\},\{5,18\},\{6,17\},\{7,16\},\{8,15\},\{9,14\},\{10,13\},\{0,2\},\{12,21\}\} \\
& \mathcal{R}_{3,1}=\{\{3,6\},\{4,7\},\{5,8\},\{1,9\},\{2,10\},\{11,14\},\{12,15\},\{16,19\},\{17,20\},\{18,21\},\{0,13\}\} \\
& \mathcal{R}_{3,2}=\{\{1,4\},\{2,5\},\{6,9\},\{7,10\},\{8,0\},\{13,16\},\{14,17\},\{15,18\},\{11,19\},\{12,20\},\{3,21\}\} \\
& \mathcal{R}_{3,3}=\{\{1,12\},\{2,11\},\{4,20\},\{5,19\},\{6,18\},\{7,17\},\{8,16\},\{9,15\},\{10,14\},\{0,3\},\{13,21\}\} \\
& \mathcal{R}_{4,1}=\{\{1,5\},\{3,7\},\{4,8\},\{6,10\},\{2,9\},\{12,16\},\{15,19\},\{17,21\},\{11,18\},\{13,20\},\{0,14\}\} \\
& \mathcal{R}_{4,2}=\{\{2,6\},\{5,9\},\{0,7\},\{1,8\},\{3,10\},\{11,15\},\{13,17\},\{14,18\},\{16,20\},\{12,19\},\{44,21\}\} \\
& \mathcal{R}_{4,3}=\{\{1,13\},\{2,12\},\{3,11\},\{5,20\},\{6,19\},\{7,18\},\{8,17\},\{9,16\},\{10,15\},\{0,4\},\{14,21\}\} \\
& \mathcal{R}_{5,1}=\{\{1,6\},\{2,7\},\{3,8\},\{4,9\},\{5,10\},\{16,21\},\{11,17\},\{12,18\},\{13,19\},\{14,20\},\{0,15\}\} \\
& \mathcal{R}_{5,2}=\{\{0,6\},\{1,7\},\{2,8\},\{3,9\},\{4,10\},\{11,16\},\{12,17\},\{13,18\},\{14,19\},\{15,20\},\{5,21\}\} \\
& \mathcal{R}_{5,3}=\{\{1,14\},\{2,13\},\{3,12\},\{4,11\},\{6,20\},\{7,19\},\{8,18\},\{9,17\},\{10,16\},\{0,5\},\{15,21\}\}
\end{aligned}
$$




$$
\mathcal{R}_{7,1}=\mathcal{C}_{8}, \mathcal{R}_{7,2}=\mathcal{C}_{9}, \mathcal{R}_{7,3}=\mathcal{C}_{10}
$$

$$
\begin{aligned}
& \mathcal{T}_{1,1}=\{\{1,2\},\{3,4\},\{5,6\},\{7,8\},\{9,10\},\{12,13\},\{14,15\},\{16,17\},\{18,19\},\{20,21\},\{0,11\}\} \\
& \mathcal{T}_{1,2}=\{\{2,3\},\{4,5\},\{6,7\},\{8,9\},\{0,10\},\{11,21\},\{13,14\},\{15,16\},\{17,18\},\{19,20\},\{1,12\}\} \\
& \mathcal{T}_{1,3}=\{\{2,13\},\{3,14\},\{4,15\},\{5,16\},\{6,17\},\{7,18\},\{8,19\},\{9,20\},\{10,21\},\{0,1\},\{11,12\}\} \\
& \mathcal{T}_{2,1}=\{\{2,4\},\{3,5\},\{6,8\},\{7,9\},\{1,10\},\{11,13\},\{14,16\},\{15,17\},\{18,20\},\{19,21\},\{0,12\}\} \\
& \mathcal{T}_{2,2}=\{\{1,3\},\{4,6\},\{5,7\},\{8,10\},\{0,9\},\{13,15\},\{16,18\},\{17,19\},\{11,20\},\{12,21\},\{2,14\}\} \\
& \mathcal{T}_{2,3}=\{\{1,13\},\{3,15\},\{4,16\},\{5,17\},\{6,18\},\{7,19\},\{8,20\},\{9,21\},\{10,11\},\{0,2\},\{12,14\}\}
\end{aligned}
$$

It is not difficult to accommodate the proofs of Lemmas 4 and 5 to all positive integers $n \equiv 1$ or $5(\bmod 6)$, where $n \geq 7$.

Lemma 6. Lemmas 4 and 5 hold for any positive integer $n \equiv 1$ or $5(\bmod 6)$, where $n \geq 7$.

Proof. When $n \equiv 1$ or $5(\bmod 6)$, the proofs for Lemmas 4 and 5 are similar, so we only show the existence of a one-factorization $\mathcal{T}$ for Lemma 5. The proof is actually similar to the case of $n$ being prime. The difference is that $\mathcal{A}_{i}$ and $\mathcal{B}_{i}$ may consist of more than one cycle if $n$ is not a prime. Nevertheless, we can use the same procedure. More precisely, we are done as follows.

Assume that we want to partition $\mathcal{A}_{i} \cup \mathcal{B}_{i} \cup \mathcal{D}_{i-1}, 1 \leq i \leq \frac{n-1}{2}$, into three one-factors. The length of the cycles generated from $\mathcal{A}_{i}$ is $\ell=\frac{n}{\operatorname{gcd}(i, n)}$ and the number of cycles is $\rho=\operatorname{gcd}(i, n)$. We denote the $j$-th cycle, $0 \leq j \leq \rho-1$, by $\mathcal{A}_{i}^{(j)}$, which consists of the following $\ell$ edges (elements are reduced to the range $\{0,1, \ldots, n-1\})$ :

$$
\{j, i+j\},\{i+j, 2 i+j\},\{2 i+j, 3 i+j\}, \ldots,\{n-i+j, j\} .
$$

For $\mathcal{B}_{i}$ we have the same $\rho$ cycles of length $\ell$, say $\mathcal{B}_{i}^{(j)}, 0 \leq j \leq \rho-1$, where the elements are reduced to the range $\{n, n+1, \ldots, 2 n-1\}$. Note that $\ell$ is odd and $\{j, i+j\} \in \mathcal{A}_{i}^{(j)},\left\{\beta_{i, j}, b_{i, j}\right\} \in \mathcal{B}_{i}^{\left(j^{\prime}\right)}$, where $\beta_{i, j} \equiv i+j-1(\bmod n), b_{i, j} \equiv 2 i+j-1(\bmod n)$ with $n \leq \beta_{i, j}, b_{i, j} \leq 2 n-1$, and $j^{\prime} \equiv j-1$ $(\bmod \rho)$ with $0 \leq j^{\prime} \leq \rho-1$. We can partition $\mathcal{A}_{i}^{(j)} \backslash\{\{j, i+j\}\}$ into two subsets $\mathcal{A}_{i, 1}^{(j)}$, and $\mathcal{A}_{i, 2}^{(j)}$ with equal size $\frac{\ell-1}{2}$ and

$$
\bigcup_{P \in \mathcal{A}_{i, 1}^{(j)}} P=\left(\bigcup_{P \in \mathcal{A}_{i}^{(j)}} P\right) \backslash\{j\}, \underset{P \in \mathcal{A}_{i, 2}^{(j)}}{\bigcup} P=\left(\bigcup_{P \in \mathcal{A}_{i}^{(j)}} P\right) \backslash\{i+j\} .
$$

Similarly, we can partition $\mathcal{B}_{i}^{\left(j^{\prime}\right)} \backslash\left\{\left\{\beta_{i, j}, b_{i, j}\right\}\right\}$ into two subsets $\mathcal{B}_{i, 1}^{\left(j^{\prime}\right)}$ and $\mathcal{B}_{i, 2}^{\left(j^{\prime}\right)}$ with equal size $\frac{\ell-1}{2}$ and

$$
\bigcup_{P \in \mathcal{B}_{i, 1}^{\left(j^{\prime}\right)}} P=\left(\bigcup_{P \in \mathcal{B}_{i}^{\left(j^{\prime}\right)}} P\right) \backslash\left\{\beta_{i, j}\right\}, \bigcup_{P \in \mathcal{B}_{i, 2}^{\left(j^{\prime}\right)}} P=\left(\bigcup_{P \in \mathcal{B}_{i}^{\left(j^{\prime}\right)}} P\right) \backslash\left\{b_{i, j}\right\} .
$$


Obviously we have $\left\{j, \beta_{i, j}\right\},\left\{i+j, b_{i, j}\right\} \in \mathcal{D}_{i-1}$. So define

$$
\begin{aligned}
\mathcal{T}_{i, 1}= & \bigcup_{j=0}^{\rho-1}\left(\mathcal{A}_{i, 1}^{(j)} \cup \mathcal{B}_{i, 1}^{\left(j^{\prime}\right)} \cup\left\{\left\{j, \beta_{i, j}\right\}\right\}\right), \\
\mathcal{T}_{i, 2}= & \bigcup_{j=0}^{\rho-1}\left(\mathcal{A}_{i, 2}^{(j)} \cup \mathcal{B}_{i, 2}^{\left(j^{\prime}\right)} \cup\left\{\left\{i+j, b_{i, j}\right\}\right\}\right), \\
\mathcal{T}_{i, 3}= & \left(\mathcal{D}_{i-1} \cup\left\{\{j, i+j\},\left\{\beta_{i, j}, b_{i, j}\right\}: 0 \leq j \leq \rho-1\right\}\right) \\
& \backslash\left\{\left\{j, \beta_{i, j}\right\},\left\{i+j, b_{i, j}\right\}: 0 \leq j \leq \rho-1\right\} .
\end{aligned}
$$

It is easy to verify that each $\mathcal{T}_{i, k}$ is a one-factor $\left(1 \leq i \leq \frac{n-1}{2}\right.$ and $\left.k=1,2,3\right)$ and Eq. (2) holds.

\subsubsection{A Latin Square for Direct Products}

This subsection is devoted only to the case when $n \equiv 5(\bmod 6)$. Let $\mathcal{R}_{i} \triangleq\left\{\mathcal{R}_{i, 1}, \mathcal{R}_{i, 2}, \mathcal{R}_{i, 3}\right\}$ and $\mathcal{T}_{i} \triangleq\left\{\mathcal{T}_{i, 1}, \mathcal{T}_{i, 2}, \mathcal{T}_{i, 3}\right\}, 1 \leq i \leq \frac{2 n-1}{3}$. The same definition for $1 \leq i \leq \frac{n-1}{2}$ is given for $n \equiv 1(\bmod 6)$. We construct a Latin square which makes a distinction between the the quadruples which are contained in the DLS Construction and those which will be constructed with the DB Construction.

Let $\nu=\frac{2 n-1}{3}$ and let $\mathcal{M}$ be a $\nu \times \nu$ Latin square defined as follows. The elements of $\mathcal{M}$ are taken from the set $\left\{\mathcal{T}_{1}, \mathcal{T}_{2}, \ldots, \mathcal{T}_{\nu}\right\}$. The $i$-th column of $\mathcal{M}$ is indexed by $\mathcal{R}_{i}, 1 \leq i \leq \nu$. The first two rows of $\mathcal{M}$ represent direct products of $\mathcal{R}_{i}$ 's by $\mathcal{T}_{j}$ 's for which some of the related quadruples are contained in the first $2 n$ PDQs of order $4 n$, and all the quadruples of the first $2 n$ PDQs are contained in these direct products, which will be explained in the next subsection. For this purpose let $\mathcal{M}\left(1, \mathcal{R}_{i}\right)=\mathcal{T}_{i}$ for $1 \leq i \leq \nu, \mathcal{M}\left(2, \mathcal{R}_{i}\right)=\mathcal{T}_{i-1}$ for $2 \leq i \leq \nu$, and $\mathcal{M}\left(2, \mathcal{R}_{1}\right)=\mathcal{T}_{\nu}$. The last $\nu-2$ rows of $\mathcal{M}$ can be chosen arbitrarily to form a Latin square. One simple choice is to take these rows as cyclic shifts of the first row.

Example 4. We continue the example for $n=11$. The first two rows of $\mathcal{M}$, represent the quadruples from configuration $(2,2)$ which are contained in the first 22 PDQs of order 44 obtained by the DLS Construction. These rows are given by

\begin{tabular}{cccccccc} 
& $\mathcal{R}_{1}$ & $\mathcal{R}_{2}$ & $\mathcal{R}_{3}$ & $\mathcal{R}_{4}$ & $\mathcal{R}_{5}$ & $\mathcal{R}_{6}$ & $\mathcal{R}_{7}$ \\
\hline 1 & $\mathcal{T}_{1}$ & $\mathcal{T}_{2}$ & $\mathcal{T}_{3}$ & $\mathcal{T}_{4}$ & $\mathcal{T}_{5}$ & $\mathcal{T}_{6}$ & $\mathcal{T}_{7}$ \\
2 & $\mathcal{T}_{7}$ & $\mathcal{T}_{1}$ & $\mathcal{T}_{2}$ & $\mathcal{T}_{3}$ & $\mathcal{T}_{4}$ & $\mathcal{T}_{5}$ & $\mathcal{T}_{6}$
\end{tabular}

These two rows can be completed to a $7 \times 7$ Latin square as follows

\begin{tabular}{cccccccc} 
& $\mathcal{R}_{1}$ & $\mathcal{R}_{2}$ & $\mathcal{R}_{3}$ & $\mathcal{R}_{4}$ & $\mathcal{R}_{5}$ & $\mathcal{R}_{6}$ & $\mathcal{R}_{7}$ \\
\hline 1 & $\mathcal{T}_{1}$ & $\mathcal{T}_{2}$ & $\mathcal{T}_{3}$ & $\mathcal{T}_{4}$ & $\mathcal{T}_{5}$ & $\mathcal{T}_{6}$ & $\mathcal{T}_{7}$ \\
2 & $\mathcal{T}_{7}$ & $\mathcal{T}_{1}$ & $\mathcal{T}_{2}$ & $\mathcal{T}_{3}$ & $\mathcal{T}_{4}$ & $\mathcal{T}_{5}$ & $\mathcal{T}_{6}$ \\
\hline 3 & $\mathcal{T}_{6}$ & $\mathcal{T}_{7}$ & $\mathcal{T}_{1}$ & $\mathcal{T}_{2}$ & $\mathcal{T}_{3}$ & $\mathcal{T}_{4}$ & $\mathcal{T}_{5}$ \\
4 & $\mathcal{T}_{5}$ & $\mathcal{T}_{6}$ & $\mathcal{T}_{7}$ & $\mathcal{T}_{1}$ & $\mathcal{T}_{2}$ & $\mathcal{T}_{3}$ & $\mathcal{T}_{4}$ \\
5 & $\mathcal{T}_{4}$ & $\mathcal{T}_{5}$ & $\mathcal{T}_{6}$ & $\mathcal{T}_{7}$ & $\mathcal{T}_{1}$ & $\mathcal{T}_{2}$ & $\mathcal{T}_{3}$ \\
6 & $\mathcal{T}_{3}$ & $\mathcal{T}_{4}$ & $\mathcal{T}_{5}$ & $\mathcal{T}_{6}$ & $\mathcal{T}_{7}$ & $\mathcal{T}_{1}$ & $\mathcal{T}_{2}$ \\
7 & $\mathcal{T}_{2}$ & $\mathcal{T}_{3}$ & $\mathcal{T}_{4}$ & $\mathcal{T}_{5}$ & $\mathcal{T}_{6}$ & $\mathcal{T}_{7}$ & $\mathcal{T}_{1}$
\end{tabular}




\subsubsection{Proof of Theorem 1}

We distinguish again between the case $n \equiv 5(\bmod 6)$ and the case $n \equiv 1(\bmod 6)$.

Case 1: $n \equiv 5(\bmod 6)$.

We continue now to conclude the second part of the construction and obtain more PDQs of order $4 n$. We construct a $(2 n-1) \times(2 n-1)$ Latin square $\mathcal{H}_{2 n-1}$ from the Latin square $\mathcal{M}$ as follows. Each row $i$ of $\mathcal{M}$ is replaced by rows $(i, 1),(i, 2)$, and $(i, 3)$, in $\mathcal{H}_{2 n-1}$. Each column $\mathcal{R}_{j}$ of $\mathcal{M}$ is replaced by columns $\mathcal{R}_{j, 1}, \mathcal{R}_{j, 2}$, and $\mathcal{R}_{j, 3}$, in $\mathcal{H}_{2 n-1}$. Any entry of $\mathcal{M}\left(i, \mathcal{R}_{j}\right)$ which is equal to $\mathcal{T}_{\ell}$ is replaced by the $3 \times 3$ Latin square in the related three rows and three columns of $\mathcal{H}_{2 n-1}$ :

\begin{tabular}{c|ccc} 
& $\mathcal{R}_{j, 1}$ & $\mathcal{R}_{j, 2}$ & $\mathcal{R}_{j, 3}$ \\
\hline$(i, 1)$ & $\mathcal{T}_{\ell, 1}$ & $\mathcal{T}_{\ell, 2}$ & $\mathcal{T}_{\ell, 3}$ \\
$(i, 2)$ & $\mathcal{T}_{\ell, 2}$ & $\mathcal{T}_{\ell, 3}$ & $\mathcal{T}_{\ell, 1}$ \\
$(i, 3)$ & $\mathcal{T}_{\ell, 3}$ & $\mathcal{T}_{\ell, 1}$ & $\mathcal{T}_{\ell, 2}$
\end{tabular}.

Then each row of $\mathcal{H}_{2 n-1}$ forms the one-factorization $\mathcal{T}$. By Lemma 2 , all the quadruples from configuration $(2,2)$ in the first $2 n$ PDQs from the DLS Construction are contained in the direct products related to the first six rows of $\mathcal{H}_{2 n-1}$. That is, the direct products of $\mathcal{R}_{j, k}$ and $\mathcal{H}_{2 n-1}\left(\left(i, k^{\prime}\right), \mathcal{R}_{j, k}\right)$ for all $1 \leq j \leq \frac{2 n-1}{3}, i=1,2$, and $1 \leq k, k^{\prime} \leq 3$. All the direct products related to the last $2 n-7$ rows of $\mathcal{H}_{2 n-1}$ are pairwise disjoint and hence by using them in the DB Construction, combined with $k$ PDQs of order $2 n$ in the DB Construction, where $k=\min \{D(2 n), 2 n-7\}$ we obtain with the first $2 n$ PDQs, a total of $2 n+\min \{D(2 n), 2 n-7\}$ PDQs of order $4 n$. Thus, we have proved Theorem 1 for $n \equiv 5(\bmod 6)$.

Example 5. Continue with the example for $n=11$. We first form the first six rows of $\mathcal{H}_{21}$ from the Latin square $\mathcal{M}$ in Example 4. If column $\mathcal{R}_{i_{1}, j_{1}}$, where $1 \leq i_{1} \leq 6$, has entry $\mathcal{T}_{i_{2}, j_{2}}$ then the $D L S$ construction has used some quadruples $\{(v, 0),(x, 1),(y, 2),(z, 3)\}$, where $\{v, x\} \in \mathcal{R}_{i_{1}, j_{1}}$ and $\{y, z\} \in \mathcal{T}_{i_{2}, j_{2}}$. Clearly, there are also many of the quadruples related to these six rows were not obtained in the first part of the construction. Finally, note that instead of some $\mathcal{R}_{i, j}$ 's and $\mathcal{T}_{i, j}$ 's we use $\mathcal{C}_{\ell}$ 's and $\mathcal{D}_{\ell}$ 's.

\begin{tabular}{|c|c|c|c|c|c|c|c|c|c|c|c|c|c|c|c|c|c|c|c|c|}
\hline $\mathcal{R}_{1,1}$ & $\mathcal{R}_{1,2}$ & $\mathcal{R}_{1,3}$ & $\mathcal{R}_{2,1}$ & $\mathcal{R}_{2,2}$ & $\mathcal{R}_{2,3}$ & $\mathcal{R}_{3,1}$ & $\mathcal{R}_{3,2}$ & $\mathcal{R}_{3,3}$ & $\mathcal{R}_{4,1}$ & $\mathcal{R}_{4,2}$ & $\mathcal{R}_{4,3}$ & $\mathcal{R}_{5,1}$ & $\mathcal{R}_{5,2}$ & $\mathcal{R}_{5,3}$ & $\mathcal{C}_{5}$ & $\mathcal{C}_{6}$ & $\mathcal{C}_{7}$ & $\mathcal{C}_{8}$ & $\mathcal{C}_{9}$ & $\mathcal{C}_{10}$ \\
\hline $\mathcal{T}_{1,1}$ & $\mathcal{T}_{1,2}$ & $\mathcal{T}_{1,3}$ & $\mathcal{T}_{2,1}$ & $\mathcal{T}_{2,2}$ & $\mathcal{T}_{2,3}$ & $\mathcal{T}_{3,1}$ & $\mathcal{T}_{3,2}$ & $\mathcal{T}_{3,3}$ & $\mathcal{T}_{4,1}$ & $\mathcal{T}_{4,2}$ & $\mathcal{T}_{4,3}$ & $\mathcal{T}_{5,1}$ & $\mathcal{T}_{5,2}$ & $\mathcal{T}_{5,3}$ & $\mathcal{D}_{5}$ & $\mathcal{D}_{6}$ & $\mathcal{D}_{7}$ & $\mathcal{D}_{8}$ & $\mathcal{D}_{9}$ & $\mathcal{D}_{10}$ \\
\hline $\mathcal{T}_{1,2}$ & $\mathcal{T}_{1,3}$ & $\mathcal{T}_{1,1}$ & $\mathcal{T}_{2,2}$ & $\mathcal{T}_{2,3}$ & $\mathcal{T}_{2,1}$ & $\mathcal{T}_{3,2}$ & $\mathcal{T}_{3,3}$ & $\mathcal{T}_{3,1}$ & $\mathcal{T}_{4,2}$ & $\mathcal{T}_{4,3}$ & $\mathcal{T}_{4,1}$ & $\mathcal{T}_{5,2}$ & $\mathcal{T}_{5,3}$ & $\mathcal{T}_{5,1}$ & $\mathcal{D}_{6}$ & $\mathcal{D}_{7}$ & $\mathcal{D}_{5}$ & $\mathcal{D}_{9}$ & $\mathcal{D}_{10}$ & $\mathcal{D}_{8}$ \\
\hline $\mathcal{T}_{1,3}$ & $\mathcal{T}_{1,1}$ & $\mathcal{T}_{1,2}$ & $\mathcal{T}_{2,3}$ & $\mathcal{T}_{2,1}$ & $\mathcal{T}_{2,2}$ & $\mathcal{T}_{3,3}$ & $\mathcal{T}_{3,1}$ & $\mathcal{T}_{3,2}$ & $\mathcal{T}_{4,3}$ & $\mathcal{T}_{4,1}$ & $\mathcal{T}_{4,2}$ & $\mathcal{T}_{5,3}$ & $\mathcal{T}_{5,1}$ & $\mathcal{T}_{5,2}$ & $\mathcal{D}_{7}$ & $\mathcal{D}_{5}$ & $\mathcal{D}_{6}$ & $\mathcal{D}_{10}$ & $\mathcal{D}_{8}$ & $\mathcal{D}_{9}$ \\
\hline $\mathcal{D}_{8}$ & $\mathcal{D}_{9}$ & $\mathcal{D}_{10}$ & $\mathcal{T}_{1,1}$ & $\mathcal{T}_{1,2}$ & $\mathcal{T}_{1,3}$ & $\mathcal{T}_{2,1}$ & $\mathcal{T}_{2,2}$ & $\mathcal{T}_{2,3}$ & $\mathcal{T}_{3,1}$ & $\mathcal{T}_{3,2}$ & $\mathcal{T}_{3,3}$ & $\mathcal{T}_{4,1}$ & $\mathcal{T}_{4,2}$ & $\mathcal{T}_{4,3}$ & $\mathcal{T}_{5,1}$ & $\mathcal{T}_{5,2}$ & $\mathcal{T}_{5,3}$ & $\mathcal{D}_{5}$ & $\mathcal{D}_{6}$ & $\mathcal{D}_{7}$ \\
\hline $\mathcal{D}_{9}$ & $\mathcal{D}_{10}$ & $\mathcal{D}_{8}$ & $\mathcal{T}_{1,2}$ & $\mathcal{T}_{1,3}$ & $\mathcal{T}_{1,1}$ & $\mathcal{T}_{2,2}$ & $\mathcal{T}_{2,3}$ & $\mathcal{T}_{2,1}$ & $\mathcal{T}_{3,2}$ & $\mathcal{T}_{3,3}$ & $\mathcal{T}_{3,1}$ & $\mathcal{T}_{4,2}$ & $\mathcal{T}_{4,3}$ & $\mathcal{T}_{4,1}$ & $\mathcal{T}_{5,2}$ & $\mathcal{T}_{5,3}$ & $\mathcal{T}_{5,1}$ & $\mathcal{D}_{6}$ & $\mathcal{D}_{7}$ & $\mathcal{D}_{5}$ \\
\hline $\mathcal{D}_{10}$ & $\mathcal{D}_{8}$ & $\mathcal{D}_{9}$ & $\mathcal{T}_{1,3}$ & $\mathcal{T}_{1,1}$ & $\mathcal{T}_{1,2}$ & $\mathcal{T}_{2,3}$ & $\mathcal{T}_{2,1}$ & $\mathcal{T}_{2,2}$ & $\mathcal{T}_{3,3}$ & $\mathcal{T}_{3,1}$ & $\mathcal{T}_{3,2}$ & $\mathcal{T}_{4,3}$ & $\mathcal{T}_{4,1}$ & $\mathcal{T}_{4,2}$ & $\mathcal{T}_{5,3}$ & $\mathcal{T}_{5,1}$ & $\mathcal{T}_{5,2}$ & $\mathcal{D}_{7}$ & $\mathcal{D}_{5}$ & $\mathcal{D}_{6}$ \\
\hline
\end{tabular}

A completed $21 \times 21$ Latin square $\mathcal{H}_{21}$ whose last 15 rows form the $15 \times 21$ Latin rectangle for the DB Construction is given next. In the first 6 rows we remove those entries for which no quadruple of the related direct product is contained in the PDQs obtained by the DLS Construction. Note, that each column contains different elements and each one of the last 15 rows contains each one-factor of $\mathcal{T}$ exactly once. 


\begin{tabular}{|c|c|c|c|c|c|c|c|c|c|c|c|c|c|}
\hline & $\mathcal{R}_{1,1}$ & $\mathcal{R}_{1,2}$ & $\mathcal{R}_{1,3}$ & & $\mathcal{R}_{5,1}$ & $\mathcal{R}_{5,2}$ & $\mathcal{R}_{5,3}$ & $\mathcal{C}_{5}$ & $\mathcal{C}_{6}$ & $\mathcal{C}_{7}$ & $\mathcal{C}_{8}$ & $\mathcal{C}_{9}$ & $\mathcal{C}_{10}$ \\
\hline 1 & $\overline{\mathcal{T}}_{1,1}$ & $\mathcal{T}_{1,2}$ & $\overline{\mathcal{T}}_{1,3}$ & & $\mathcal{T}_{5,1}$ & $\mathcal{T}_{5,2}$ & $\mathcal{T}_{5,3}$ & $\mathcal{D}_{5}$ & $\overline{\mathcal{D}}_{6}$ & $\overline{\mathcal{D}_{7}}$ & $\overline{\mathcal{D}_{8}}$ & $\overline{\mathcal{D}_{9}}$ & $\overline{\mathcal{D}}_{10}$ \\
\hline 2 & $\mathcal{T}_{1,2}$ & $\mathcal{T}_{1,3}$ & $\mathcal{T}_{1,1}$ & $\cdots$ & $\mathcal{T}_{5,2}$ & $\mathcal{T}_{5,3}$ & $\mathcal{T}_{5,1}$ & & & & & & \\
\hline 3 & $\mathcal{T}_{1,3}$ & $\mathcal{T}_{1,1}$ & $\mathcal{T}_{1,2}$ & . & $\mathcal{T}_{5,3}$ & $\mathcal{T}_{5,1}$ & $\mathcal{T}_{5,2}$ & & $\mathcal{D}_{5}$ & $\mathcal{D}_{6}$ & & $\mathcal{D}_{8}$ & $\mathcal{D}_{9}$ \\
\hline 4 & & & $\mathcal{D}_{10}$ & & $\mathcal{T}_{4,1}$ & $\mathcal{T}_{4,2}$ & $\mathcal{T}_{4,3}$ & $\mathcal{T}_{5,1}$ & & & & & \\
\hline 5 & & $\mathcal{D}_{10}$ & & $\cdots$ & $\mathcal{T}_{4,2}$ & $\mathcal{T}_{4,3}$ & $\mathcal{T}_{4,1}$ & $\mathcal{T}_{5,2}$ & & & & & \\
\hline 6 & $\mathcal{D}_{10}$ & & & $\cdots$ & $\mathcal{T}_{4,3}$ & $\mathcal{T}_{4,1}$ & $\mathcal{T}_{4,2}$ & $\mathcal{T}_{5,3}$ & & & $\mathcal{D}_{7}$ & & \\
\hline 7 & $\mathcal{D}_{5}$ & $\mathcal{D}_{6}$ & $\mathcal{D}_{7}$ & & $\mathcal{T}_{3,1}$ & $\mathcal{T}_{3,2}$ & $\overline{\mathcal{T}}_{3,3}$ & $\mathcal{T}_{4,1}$ & $\mathcal{T}_{4,2}$ & $\mathcal{T}_{4,3}$ & $\mathcal{T}_{5,1}$ & $\mathcal{T}_{5,2}$ & $\mathcal{T}_{5,3}$ \\
\hline 8 & $\mathcal{D}_{6}$ & $\mathcal{D}_{7}$ & $\mathcal{D}_{5}$ & . & $\mathcal{T}_{3,2}$ & $\mathcal{T}_{3,3}$ & $\mathcal{T}_{3,1}$ & $\mathcal{T}_{4,2}$ & $\mathcal{T}_{4,3}$ & $\mathcal{T}_{4,1}$ & $\mathcal{T}_{5,2}$ & $\mathcal{T}_{5,3}$ & $\mathcal{T}_{5,1}$ \\
\hline 9 & $\mathcal{D}_{7}$ & $\mathcal{D}_{5}$ & $\mathcal{D}_{6}$ & $\cdots$ & $\mathcal{T}_{3,3}$ & $\mathcal{T}_{3,1}$ & $\mathcal{T}_{3,2}$ & $\mathcal{T}_{4,3}$ & $\mathcal{T}_{4,1}$ & $\mathcal{T}_{4,2}$ & $\mathcal{T}_{5,3}$ & $\mathcal{T}_{5,1}$ & $\mathcal{T}_{5,2}$ \\
\hline 10 & $\mathcal{T}_{5,1}$ & $\mathcal{T}_{5,2}$ & $\mathcal{T}_{5,3}$ & 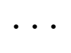 & $\mathcal{T}_{2,1}$ & $\mathcal{T}_{2,2}$ & $\mathcal{T}_{2,3}$ & $\mathcal{T}_{3,1}$ & $\mathcal{T}_{3,2}$ & $\mathcal{T}_{3,3}$ & $\mathcal{T}_{4,1}$ & $\mathcal{T}_{4,2}$ & $\mathcal{T}_{4,3}$ \\
\hline 11 & $\mathcal{T}_{5,2}$ & $\mathcal{T}_{5,3}$ & $\mathcal{T}_{5,1}$ & . & $\mathcal{T}_{2,2}$ & $\mathcal{T}_{2,3}$ & $\mathcal{T}_{2,1}$ & $\mathcal{T}_{3,2}$ & $\mathcal{T}_{3,3}$ & $\mathcal{T}_{3,1}$ & $\mathcal{T}_{4,2}$ & $\mathcal{T}_{4,3}$ & $\mathcal{T}_{4,1}$ \\
\hline 12 & $\mathcal{T}_{5,3}$ & $\mathcal{T}_{5,1}$ & $\mathcal{T}_{5,2}$ & . & $\mathcal{T}_{2,3}$ & $\mathcal{T}_{2,1}$ & $\mathcal{T}_{2,2}$ & $\mathcal{T}_{3,3}$ & $\mathcal{T}_{3,1}$ & $\mathcal{T}_{3,2}$ & $\mathcal{T}_{4,3}$ & $\mathcal{T}_{4,1}$ & $\mathcal{T}_{4,2}$ \\
\hline 13 & $\mathcal{T}_{4,1}$ & $\mathcal{T}_{4,2}$ & $\mathcal{T}_{4,3}$ & $\cdots$ & $\mathcal{T}_{1,1}$ & $\mathcal{T}_{1,2}$ & $\mathcal{T}_{1,3}$ & $\mathcal{T}_{2,1}$ & $\mathcal{T}_{2,2}$ & $\mathcal{T}_{2,3}$ & $\mathcal{T}_{3,1}$ & $\mathcal{T}_{3,2}$ & $\mathcal{T}_{3,3}$ \\
\hline 14 & $\mathcal{T}_{4,2}$ & $\mathcal{T}_{4,3}$ & $\mathcal{T}_{4,1}$ & $\ldots$ & $\mathcal{T}_{1,2}$ & $\mathcal{T}_{1,3}$ & $\mathcal{T}_{1,1}$ & $\mathcal{T}_{2,2}$ & $\mathcal{T}_{2,3}$ & $\mathcal{T}_{2,1}$ & $\mathcal{T}_{3,2}$ & $\mathcal{T}_{3,3}$ & $\mathcal{T}_{3,1}$ \\
\hline 15 & $\mathcal{T}_{4,3}$ & $\mathcal{T}_{4,1}$ & $\mathcal{T}_{4,2}$ & - & $\mathcal{T}_{1,3}$ & $\mathcal{T}_{1,1}$ & $\mathcal{T}_{1,2}$ & $\mathcal{T}_{2,3}$ & $\mathcal{T}_{2,1}$ & $\mathcal{T}_{2,2}$ & $\mathcal{T}_{3,3}$ & $\mathcal{T}_{3,1}$ & $\mathcal{T}_{3,2}$ \\
\hline 16 & $\mathcal{T}_{3,1}$ & $\mathcal{T}_{3,2}$ & $\mathcal{T}_{3,3}$ & . & $\mathcal{D}_{8}$ & $\mathcal{D}_{9}$ & $\mathcal{D}_{10}$ & $\mathcal{T}_{1,1}$ & $\mathcal{T}_{1,2}$ & $\mathcal{T}_{1,3}$ & $\mathcal{T}_{2,1}$ & $\mathcal{T}_{2,2}$ & $\mathcal{T}_{2,3}$ \\
\hline 17 & $\mathcal{T}_{3,2}$ & $\mathcal{T}_{3,3}$ & $\mathcal{T}_{3,1}$ & 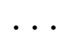 & $\mathcal{D}_{9}$ & $\mathcal{D}_{10}$ & $\mathcal{D}_{8}$ & $\mathcal{T}_{1,2}$ & $\mathcal{T}_{1,3}$ & $\mathcal{T}_{1,1}$ & $\mathcal{T}_{2,2}$ & $\mathcal{T}_{2,3}$ & $\mathcal{T}_{2,1}$ \\
\hline 18 & $\mathcal{T}_{3,3}$ & $\mathcal{T}_{3,1}$ & $\mathcal{T}_{3,2}$ & . & $\mathcal{D}_{10}$ & $\mathcal{D}_{8}$ & $\mathcal{D}_{9}$ & $\mathcal{T}_{1,3}$ & $\mathcal{T}_{1,1}$ & $\mathcal{T}_{1,2}$ & $\mathcal{T}_{2,3}$ & $\mathcal{T}_{2,1}$ & $\mathcal{T}_{2,2}$ \\
\hline 19 & $\mathcal{T}_{2,1}$ & $\mathcal{T}_{2,2}$ & $\mathcal{T}_{2,3}$ & . & $\mathcal{D}_{5}$ & $\mathcal{D}_{6}$ & $\mathcal{D}_{7}$ & $\mathcal{D}_{8}$ & $\mathcal{D}_{9}$ & $\mathcal{D}_{10}$ & $\mathcal{T}_{1,1}$ & $\mathcal{T}_{1,2}$ & $\mathcal{T}_{1,3}$ \\
\hline 20 & $\mathcal{T}_{2,2}$ & $\mathcal{T}_{2,3}$ & $\mathcal{T}_{2,1}$ & - & $\mathcal{D}_{6}$ & $\mathcal{D}_{7}$ & $\mathcal{D}_{5}$ & $\mathcal{D}_{9}$ & $\mathcal{D}_{10}$ & $\mathcal{D}_{8}$ & $\mathcal{T}_{1,2}$ & $\mathcal{T}_{1,3}$ & $\mathcal{T}_{1,1}$ \\
\hline 21 & $\mathcal{T}_{2,3}$ & $\mathcal{T}_{2,1}$ & $\mathcal{T}_{2,2}$ & . & $\mathcal{D}_{7}$ & $\mathcal{D}_{5}$ & $\mathcal{D}_{6}$ & $\mathcal{D}_{10}$ & $\mathcal{D}_{8}$ & $\mathcal{D}_{9}$ & $\mathcal{T}_{1,3}$ & $\mathcal{T}_{1,1}$ & $\mathcal{T}_{1,2}$ \\
\hline
\end{tabular}

Case $2: n \equiv 1(\bmod 6)$.

In this case the direct products are not obtained in a straightforward way like in Case 1 for $n \equiv 5(\bmod 6)$. But, the general method is very similar. We start with an example for $n=7$.

Example 6. Continue with the example for $n=7$. The last seven rows of the following array form one of the possible direct products to produce seven sets of quadruples from configuration $(2,2)$ for the DB Construction. Taking the direct products of the column labels and the corresponding entries in the first six rows contains all the quadruples obtained from the DLS Construction (and also some other quadruples).

\begin{tabular}{|c|c|c|c|c|c|c|c|c|c|c|c|c|}
\hline $\mathcal{R}_{1,1}$ & $\mathcal{R}_{1,2}$ & $\mathcal{R}_{1,3}$ & $\mathcal{R}_{2,1}$ & $\mathcal{R}_{2,2}$ & $\mathcal{R}_{2,3}$ & $\mathcal{R}_{3,1}$ & $\mathcal{R}_{3,2}$ & $\mathcal{R}_{3,3}$ & $\mathcal{C}_{3}$ & $\mathcal{C}_{4}$ & $\mathcal{C}_{5}$ & $\mathcal{C}_{6}$ \\
\hline $\mathcal{T}_{1,1}$ & $\mathcal{T}_{1,2}$ & $\mathcal{T}_{1,3}$ & $\mathcal{T}_{2,1}$ & $\mathcal{T}_{2,2}$ & $\mathcal{T}_{2,3}$ & $\mathcal{T}_{3,1}$ & $\mathcal{T}_{3,2}$ & $\mathcal{T}_{3,3}$ & $\overline{\mathcal{D}}_{3}$ & $\mathcal{D}_{4}$ & $\mathcal{D}_{5}$ & $\mathcal{D}_{6}$ \\
\hline $\mathcal{T}_{1,2}$ & $\mathcal{T}_{1,3}$ & $\mathcal{T}_{1,1}$ & $\mathcal{T}_{2,2}$ & $\mathcal{T}_{2,3}$ & $\mathcal{T}_{2,1}$ & $\mathcal{T}_{3,2}$ & $\mathcal{T}_{3,3}$ & $\mathcal{T}_{3,1}$ & & $\mathcal{D}_{3}$ & $\mathcal{D}_{4}$ & $\mathcal{D}_{5}$ \\
\hline $\mathcal{T}_{1,3}$ & $\mathcal{T}_{1,1}$ & $\mathcal{T}_{1,2}$ & $\mathcal{T}_{2,3}$ & $\mathcal{T}_{2,1}$ & $\mathcal{T}_{2,2}$ & $\mathcal{T}_{3,3}$ & $\mathcal{T}_{3,1}$ & $\mathcal{T}_{3,2}$ & & & & \\
\hline $\mathcal{D}_{6}$ & & & $\mathcal{T}_{1,1}$ & $\mathcal{T}_{1,2}$ & $\mathcal{T}_{1,3}$ & $\mathcal{T}_{2,1}$ & $\mathcal{T}_{2,2}$ & $\mathcal{T}_{2,3}$ & $\mathcal{T}_{3,1}$ & & & \\
\hline & $\mathcal{D}_{6}$ & & $\mathcal{T}_{1,2}$ & $\mathcal{T}_{1,3}$ & $\mathcal{T}_{1,1}$ & $\mathcal{T}_{2,2}$ & $\mathcal{T}_{2,3}$ & $\mathcal{T}_{2,1}$ & $\mathcal{T}_{3,2}$ & & & \\
\hline & & $\mathcal{D}_{6}$ & $\mathcal{T}_{1,3}$ & $\mathcal{T}_{1,1}$ & $\mathcal{T}_{1,2}$ & $\mathcal{T}_{2,3}$ & $\mathcal{T}_{2,1}$ & $\mathcal{T}_{2,2}$ & $\mathcal{T}_{3,3}$ & & & \\
\hline $\mathcal{T}_{3,1}$ & $\mathcal{T}_{3,2}$ & $\mathcal{T}_{3,3}$ & $\mathcal{D}_{3}$ & $\mathcal{D}_{4}$ & $\mathcal{D}_{5}$ & $\mathcal{D}_{6}$ & $\mathcal{T}_{1,2}$ & $\mathcal{T}_{1,3}$ & $\mathcal{T}_{2,3}$ & $\mathcal{T}_{2,2}$ & $\mathcal{T}_{2,1}$ & $\mathcal{T}_{1,1}$ \\
\hline $\mathcal{T}_{3,2}$ & $\mathcal{T}_{3,3}$ & $\mathcal{T}_{3,1}$ & $\mathcal{D}_{4}$ & $\mathcal{D}_{5}$ & $\mathcal{D}_{3}$ & $\mathcal{T}_{1,2}$ & $\mathcal{D}_{6}$ & $\mathcal{T}_{1,1}$ & $\mathcal{T}_{2,2}$ & $\mathcal{T}_{1,3}$ & $\mathcal{T}_{2,3}$ & $\mathcal{T}_{2,1}$ \\
\hline $\mathcal{T}_{3,3}$ & $\mathcal{T}_{3,1}$ & $\mathcal{T}_{3,2}$ & $\mathcal{D}_{5}$ & $\mathcal{D}_{3}$ & $\mathcal{D}_{4}$ & $\mathcal{T}_{1,3}$ & $\mathcal{T}_{1,1}$ & $\mathcal{D}_{6}$ & $\mathcal{T}_{2,1}$ & $\mathcal{T}_{2,3}$ & $\mathcal{T}_{1,2}$ & $\mathcal{T}_{2,2}$ \\
\hline $\mathcal{T}_{2,1}$ & $\mathcal{T}_{2,2}$ & $\mathcal{T}_{2,3}$ & $\mathcal{D}_{6}$ & $\mathcal{T}_{3,2}$ & $\mathcal{T}_{3,3}$ & $\mathcal{D}_{3}$ & $\mathcal{D}_{4}$ & $\mathcal{D}_{5}$ & $\mathcal{T}_{1,1}$ & $\mathcal{T}_{1,2}$ & $\mathcal{T}_{1,3}$ & $\mathcal{T}_{3,1}$ \\
\hline $\mathcal{T}_{2,2}$ & $\mathcal{T}_{2,3}$ & $\mathcal{T}_{2,1}$ & $\mathcal{T}_{3,2}$ & $\mathcal{D}_{6}$ & $\mathcal{T}_{3,1}$ & $\mathcal{D}_{4}$ & $\mathcal{D}_{5}$ & $\mathcal{D}_{3}$ & $\mathcal{T}_{1,2}$ & $\mathcal{T}_{1,1}$ & $\mathcal{T}_{3,3}$ & $\mathcal{T}_{1,3}$ \\
\hline $\mathcal{T}_{2,3}$ & $\mathcal{T}_{2,1}$ & $\mathcal{T}_{2,2}$ & $\mathcal{T}_{3,3}$ & $\mathcal{T}_{3,1}$ & $\mathcal{D}_{6}$ & $\mathcal{D}_{5}$ & $\mathcal{D}_{3}$ & $\mathcal{D}_{4}$ & $\mathcal{T}_{1,3}$ & $\mathcal{T}_{3,2}$ & $\mathcal{T}_{1,1}$ & $\mathcal{T}_{1,2}$ \\
\hline $\mathcal{D}_{3}$ & $\mathcal{D}_{4}$ & $\mathcal{D}_{5}$ & $\mathcal{T}_{3,1}$ & $\mathcal{T}_{3,3}$ & $\mathcal{T}_{3,2}$ & $\mathcal{T}_{1,1}$ & $\mathcal{T}_{1,3}$ & $\mathcal{T}_{1,2}$ & $\mathcal{D}_{6}$ & $\mathcal{T}_{2,1}$ & $\mathcal{T}_{2,2}$ & $\mathcal{T}_{2,3}$ \\
\hline
\end{tabular}


If $n>7$ consider a $(2 n-1) \times(2 n-1)$ array $\mathcal{H}_{2 n-1}(\mathcal{H}$ in short $)$ whose first $\frac{3 n-3}{2}$ columns are indexed by $\mathcal{R}_{i, j}, 1 \leq i \leq \frac{n-1}{2}, 1 \leq j \leq 3$, and whose last $\frac{n+1}{2}$ columns are indexed by $\mathcal{C}_{i}$, $\frac{n-1}{2} \leq i \leq n-1$. The entries of the array are the elements $\mathcal{T}_{i, j}, 1 \leq i \leq \frac{n-1}{2}, 1 \leq j \leq 3$, and $\mathcal{D}_{i}, \frac{n-1}{2} \leq i \leq n-1$. The first $\frac{3 n-3}{2}$ columns of the first six rows of $\mathcal{H}$ are defined by abused of definition similar for $\mathcal{H}_{2 n-1}$, in the case of $n \equiv 5(\bmod 6)$. That is, we choose the first two rows and the first $\frac{n-1}{2}$ columns of the Latin square $\mathcal{M}$ and substitute $\mathcal{R}_{i}$ by $\mathcal{R}_{i, 1}, \mathcal{R}_{i, 2}, \mathcal{R}_{i, 3}$, and replace every entry $\mathcal{T}_{\ell}$ with a Latin square with elements $\mathcal{T}_{\ell, 1}, \mathcal{T}_{\ell, 2}, \mathcal{T}_{l, 3}$, but note that the first three columns of the resultant rows 4,5 , and 6 , form a $3 \times 3$ Latin square with the elements $\mathcal{D}_{n-3}, \mathcal{D}_{n-2}$, $\mathcal{D}_{n-1}$. The other entries of the first six rows of $\mathcal{H}$ are defined as follows.

- $\mathcal{H}\left(1, \mathcal{C}_{i}\right)=\mathcal{D}_{i}, \frac{n-1}{2} \leq i \leq n-1$.

- $\mathcal{H}\left(2, \mathcal{C}_{i}\right)=\mathcal{D}_{i-1}, \frac{n+1}{2} \leq i \leq n-1$, and $\left.\mathcal{H}\left(2, \mathcal{C}_{(n-1) / 2}\right)\right)=\mathcal{D}_{n-1}$.

- $\left.\mathcal{H}\left(3, \mathcal{C}_{i}\right)=\mathcal{D}_{i-2}, \frac{n+3}{2} \leq i \leq n-1, \mathcal{H}\left(3, \mathcal{C}_{(n-1) / 2}\right)\right)=\mathcal{D}_{n-2}$, and $\left.\mathcal{H}\left(3, \mathcal{C}_{(n+1) / 2}\right)\right)=\mathcal{D}_{n-1}$.

- Columns $\mathcal{C}_{(n-1) / 2}, \mathcal{C}_{(n+1) / 2}, \mathcal{C}_{(n+3) / 2}$, in rows 4,5 , and 6 , form a $3 \times 3$ Latin square with the elements $\mathcal{T}_{(n-1) / 2, j}, 1 \leq j \leq 3$.

- $\mathcal{H}\left(4, \mathcal{C}_{i}\right)=\mathcal{D}_{i-3}, \frac{n+5}{2} \leq i \leq n-1$.

- $\mathcal{H}\left(5, \mathcal{C}_{i}\right)=\mathcal{D}_{i-4}, \frac{n+7}{2} \leq i \leq n-1$, and $\left.\mathcal{H}\left(5, \mathcal{C}_{(n+5) / 2}\right)\right)=\mathcal{D}_{n-4}$.

- $\left.\mathcal{H}\left(6, \mathcal{C}_{i}\right)=\mathcal{D}_{i-5}, \frac{n+9}{2} \leq i \leq n-1, \mathcal{H}\left(6, \mathcal{C}_{(n+5) / 2}\right)\right)=\mathcal{D}_{n-5}$, and $\left.\mathcal{H}\left(6, \mathcal{C}_{(n+7) / 2}\right)\right)=\mathcal{D}_{n-4}$.

One can easily verifies that these six rows form a $6 \times(2 n-1)$ Latin rectangle. It can be completed to the $(2 n-1) \times(2 n-1)$ Latin square $\mathcal{H}$, whose last $2 n-7$ rows define the disjoint quadruples from configuration $(2,2)$ for the DB Construction. Thus, we have proved Theorem 1 for $n \equiv 1(\bmod 6)$.

Example 7. The six rows of the Latin rectangle for $n=19$ is given in the following array.

\begin{tabular}{cccccccccccccccccc}
$\mathcal{R}_{1,1}$ & $\mathcal{R}_{1,2}$ & $\mathcal{R}_{1,3}$ & $\mathcal{R}_{2,1}$ & $\mathcal{R}_{2,2}$ & $\mathcal{R}_{2,3}$ & $\ldots$ & $\mathcal{R}_{9,1}$ & $\mathcal{R}_{9,2}$ & $\mathcal{R}_{9,3}$ & $\mathcal{C}_{9}$ & $\mathcal{C}_{10}$ & $\mathcal{C}_{11}$ & $\mathcal{C}_{12}$ & $\mathcal{C}_{13}$ & $\cdots$ & $\mathcal{C}_{17}$ & $\mathcal{C}_{18}$ \\
\hline $\mathcal{T}_{1,1}$ & $\mathcal{T}_{1,2}$ & $\mathcal{T}_{1,3}$ & $\mathcal{T}_{2,1}$ & $\mathcal{T}_{2,2}$ & $\mathcal{T}_{2,3}$ & $\cdots$ & $\mathcal{T}_{9,1}$ & $\mathcal{T}_{9,2}$ & $\mathcal{T}_{9,3}$ & $\mathcal{D}_{9}$ & $\mathcal{D}_{10}$ & $\mathcal{D}_{11}$ & $\mathcal{D}_{12}$ & $\mathcal{D}_{13}$ & $\cdots$ & $\mathcal{D}_{17}$ & $\mathcal{D}_{18}$ \\
$\mathcal{T}_{1,2}$ & $\mathcal{T}_{1,3}$ & $\mathcal{T}_{1,1}$ & $\mathcal{T}_{2,2}$ & $\mathcal{T}_{2,3}$ & $\mathcal{T}_{2,1}$ & $\ldots$ & $\mathcal{T}_{9,2}$ & $\mathcal{T}_{9,3}$ & $\mathcal{T}_{9,1}$ & $\mathcal{D}_{18}$ & $\mathcal{D}_{9}$ & $\mathcal{D}_{10}$ & $\mathcal{D}_{11}$ & $\mathcal{D}_{12}$ & $\cdots$ & $\mathcal{D}_{16}$ & $\mathcal{D}_{17}$ \\
$\mathcal{T}_{1,3}$ & $\mathcal{T}_{1,1}$ & $\mathcal{T}_{1,2}$ & $\mathcal{T}_{2,3}$ & $\mathcal{T}_{2,1}$ & $\mathcal{T}_{2,2}$ & $\ldots$ & $\mathcal{T}_{9,3}$ & $\mathcal{T}_{9,1}$ & $\mathcal{T}_{9,2}$ & $\mathcal{D}_{17}$ & $\mathcal{D}_{18}$ & $\mathcal{D}_{9}$ & $\mathcal{D}_{10}$ & $\mathcal{D}_{11}$ & $\cdots$ & $\mathcal{D}_{15}$ & $\mathcal{D}_{16}$ \\
$\mathcal{D}_{16}$ & $\mathcal{D}_{17}$ & $\mathcal{D}_{18}$ & $\mathcal{T}_{1,1}$ & $\mathcal{T}_{1,2}$ & $\mathcal{T}_{1,3}$ & $\ldots$ & $\mathcal{T}_{8,1}$ & $\mathcal{T}_{8,2}$ & $\mathcal{T}_{8,3}$ & $\mathcal{T}_{9,1}$ & $\mathcal{T}_{9,2}$ & $\mathcal{T}_{9,3}$ & $\mathcal{D}_{9}$ & $\mathcal{D}_{10}$ & $\cdots$ & $\mathcal{D}_{14}$ & $\mathcal{D}_{15}$ \\
$\mathcal{D}_{17}$ & $\mathcal{D}_{18}$ & $\mathcal{D}_{16}$ & $\mathcal{T}_{1,2}$ & $\mathcal{T}_{1,3}$ & $\mathcal{T}_{1,1}$ & $\ldots$ & $\mathcal{T}_{8,2}$ & $\mathcal{T}_{8,3}$ & $\mathcal{T}_{8,1}$ & $\mathcal{T}_{9,2}$ & $\mathcal{T}_{9,3}$ & $\mathcal{T}_{9,1}$ & $\mathcal{D}_{15}$ & $\mathcal{D}_{9}$ & $\cdots$ & $\mathcal{D}_{13}$ & $\mathcal{D}_{14}$ \\
$\mathcal{D}_{18}$ & $\mathcal{D}_{16}$ & $\mathcal{D}_{17}$ & $\mathcal{T}_{1,3}$ & $\mathcal{T}_{1,1}$ & $\mathcal{T}_{1,2}$ & $\ldots$ & $\mathcal{T}_{8,3}$ & $\mathcal{T}_{8,1}$ & $\mathcal{T}_{8,2}$ & $\mathcal{T}_{9,3}$ & $\mathcal{T}_{9,1}$ & $\mathcal{T}_{9,2}$ & $\mathcal{D}_{14}$ & $\mathcal{D}_{15}$ & $\cdots$ & $\mathcal{D}_{12}$ & $\mathcal{D}_{13}$
\end{tabular}

Finally, note that we have solved the case where $n \equiv 5(\bmod 6)$ in exactly the same way as the case $n \equiv 1(\bmod 6)$. The advantage of the presented solution for $n \equiv 5(\bmod 6)$ is that it provides an explicit construction of the Latin square $\mathcal{H}_{2 n-1}$ from a Latin square $\mathcal{M}$ of order $\frac{2 n-1}{3}$.

\section{Conclusion, Discussion, and Future Research}

We have presented a recursive construction for PDQs of order $4 n$ from a set of PDQs or order $2 n$, where $n$ is congruent to 1 or 5 modulo 6 . Having almost a large set of PDQs order $2 n$ the 
construction would yield an almost large set of PDQs order $4 n$. More precisely, if $n>5$ is an odd integer not divisible by 3 , then

$$
D(4 n) \geq 2 n+\min \{D(2 n), 2 n-7\} .
$$

The first question that one might ask is whether the recursive formula can be improved to $D(4 n)=2 n+\min \{D(2 n), 2 n-3\}$ by partitioning the unused quadruples of configuration $(2,2)$ into four sets, each one of size $n^{2}(2 n-1)$, in which no triple is contained in more than one quadruple. Unfortunately, the answer is negative. To see that one can easily verify that all the quadruples of the form

$$
\{(v, 0),(x, 0),(y, 1),(z, 1)\}, \quad\{v, x\} \subset \mathcal{A}_{1} \cup \mathcal{B}_{1} \cup \mathcal{A}_{2} \cup \mathcal{B}_{2}, \quad\{y, z\} \subset \mathcal{D}_{0}
$$

are not used in our construction, which implies that $\mathcal{A}_{1} \cup \mathcal{B}_{1} \cup \mathcal{A}_{2} \cup \mathcal{B}_{2}$ should be partitioned into four one-factors of $\mathbb{Z}_{2 n}$ which is obviously impossible.

Next, we would like to know how the main construction is compared to previous results. In [1] we have the recursive formula

$$
D(4 n) \geq 2 n+\min \{D(2 n), n-2\} \text { for } n \equiv 1 \text { or } 5(\bmod 6)
$$

which is clearly inferior to the new result. An interesting comparison is with the lower bound on $D(n)$ given in [3]. For $n \equiv 1$ or $5(\bmod 6)$, it is proved that $D(4 n) \geq 3 r$ if there exists a set of $r$ mutually 2-chromatic PDQs of order $2 n$. When $r=n$ we have that the set of $3 n$ PDQs is maximal, in the sense that the set cannot be extended to contain more PDQs. Our new result is better. If there exists a set of $r$ PDQs of order $2 n$, Theorem 1 implies that $D(4 n) \geq 2 n+r$ and if $r=n$ then the two results coincide, but while the set of PDQs in [3] is maximal, our set might be extended. In fact, if $D(2 n)>n$, then Theorem 1 implies $D(4 n)>3 n$, while the construction in [3] cannot be applied since in this case the PDQs of order $2 n$ cannot be mutually 2-chromatic.

There are many open questions which are remained to be answered in the future. We list some of them in order of difficulty, from the most difficult one to the easiest one to our opinion.

1. Construct an infinite family of large sets of PDQs of order $n$. We conjecture that $n=2^{m}$, $m \geq 4$, should be the easiest case, if there is an easy one.

2. Construct one nontrivial large set of SQSs of order $n$. We conjecture that $n=16$ should be the easiest target.

3. Prove that $D(2 n) \geq n+D(n)$ for $n \equiv 2$ or $4(\bmod 6)$.

4. Prove that $D(2 n) \geq n+D(n)$ for an infinite sequence of values of $n$.

5. Prove that $D(2 n) \geq n$ for all $n \equiv 1$ or $2(\bmod 3)$ and $n>4$. Note that this holds for $n \equiv 2$ or $4(\bmod 6)$.

6. Improve Theorem 1 for all $n \equiv 2$ or $4(\bmod 6)$. 


\section{References}

[1] T. Etzion, Partitions for quadruples, Ars Combinatoria, 36 (1993), 296 - 308.

[2] T. Etzion, On threshold schemes from large sets, Journal of Combinatorial Designs, 4 (1996), $323-338$.

[3] T. Etzion and A. Hartman, Toward a large set of Steiner quadruple systems, SIAM Journal on Discrete Mathematics, 4 (1991), 182 - 195.

[4] P. Hall, On representatives of subsets, J. London Math. Society, 10 (1935), 26 - 30.

[5] H. Hanani, On quadruple systems, Canad. J. Math., 12 (1960), 145 - 157.

[6] L. Ji, A new existence proof for large sets of disjoint Steiner triple systems, J. Combinatorial Theory, Series A, 112 (2005), 308 - 327.

[7] P. Keevash, The existence of designs II, arxiv.org/abs/1802.05900, February 2018.

[8] A. Kotzig, K. Lindner, And A. Rosa, Latin squares with no subsquares of order two and disjoint Steiner triple systems, Utilias Mathematica, 7 (1975), 287 - 294.

[9] E. S. Kramer and D. M. Mesner, Intersections among Steiner systems, J. Combinatorial Theory, Series A, 16 (1974), $273-285$.

[10] K. Lindner, A note on disjoint Steiner quadruple systems, Ars Combinatoria, 3 (1977), 271 -276 .

[11] K. Lindner, On the construction of pairwise disjoint Steiner quadruple systems, Ars Combinatoria, 19 (1985), 153 - 156.

[12] J. X. Lu, On large sets of disjoint Steiner triple systems, I - III, J. Combinatorial Theory, Series A, 34 (1983), 140 - 146, 147 - 155, 156 - 182.

[13] J. X. Lu, On large sets of disjoint Steiner triple systems, IV - VI, J. Combinatorial Theory, Series A, 37 (1984), 136 - 163, 164 - 188, 189 - 192.

[14] K. T. Phelps, A class of 2-chromatic SQS(22), Disc. Math., 97 (1991), 333 - 338.

[15] K. T. Phelps And A. Rosa, 2-Chromatic Steiner quadruple systems, European J. Combin., 1 (1980), $253-258$.

[16] L. Teirlinck, A completion of Lu's determination of the spectrum for large sets of disjoint Steiner triple systems, J. Combinatorial Theory, Series A, 57 (1991), $302-305$.

[17] W. D. Wallis, One-factorizations, Kluwer Academic Publisher, 1997. 